\title{
De l'application de la perméabilité à l'air du sol en agronomie II. Intérêt de la perméabilité à l'air pour la caractérisation de la structure du sol et des modalités d'enracinement
}

\author{
H. Mettauer et P. Hinsinger \\ INRA Station d'Agronomie, 28, rue de Herrlesheim, 68021 Colmar, France
}

(reçu le 21-12-1987, accepté le 16-10-1988)

Résumé - Après avoir présenté dans un premier article les fondements théoriques de la perméabilité à l'air, les auteurs montrent dans ce second article l'intérêt de cette grandeur pour une caractérisation rapide et peu destructive de la structure du sol.

Dans une première partie, ils définissent un «cahier des charges» pour une appréciation rapide de la structure compte tenu des limites des méthodes existantes.

Dans une seconde partie, ils traitent de l'intérêt d'ordre conceptuel de la mesure de la perméabilité à l'air pour une appréciation agronomique de la structure en termes de circulation des fluides, de porosité continue et de colonisation racinaire.

Dans une dernière partie, les auteurs développent l'intérêt technique de cette méthode de mesure pour rendre compte des variabilités spatiale (horizontale et verticale) et temporelle de la structure. Les principales références bibliographiques sont rappelées.

perméabilité à l'air - mesures - sol - structure - enracinement

Summary - Air permeability of soils and its application to agronomy. II. Benefit of the air permeability for characterization of soil structure and root colonization. In a first article, the authors presented the theorical basis of air permeability. In this one, they show the benefit of this method for a rapid and non-destructive characterization of the soil structure.

In the first part, they specify the necessary parameters for a rapid appreciation of this structure, taking into account the technical limits of the existing methods.

In the second part, they deal with the conceptual interest of the air permeability measure for an agronomic appreciation of the structure in terms of fluid circulation and continuous porosity and in terms of root colonization.

In the last part, the authors develop the technical interest of this method to account for the spatial (horizontal and vertical) and temporal variabilities of the soil structure. They also cite the main bibliographical references.

air permeability - measures - soil - structure - root system

\section{Introduction}

La structure du sol apparaît comme une condition de production déterminante, en agissant sur la plante au travers de divers facteurs physiques : humidité, aération, température et résistance mécanique (Russel, 1949; Eavis, 1972). Ceux-ci interviennent, principalement sur la croissance et le développement des organes végétaux présents dans le sol, voire, indirectement, sur la plante entière au travers de facteurs tro- phiques (interaction de la structure du sol avec la disponibilité des éléments et de l'eau du sol ainsi qu'avec son chimisme) (Blanchet et al., 1972). En fait, au-delà des premiers stades phénologiques pendant lesquels la structure induit des répercussions sur les plantes entières (effet de la structure du lit de semence sur l'humectation des graines et leur germination, effet de la battance sur la levée des plantules), c'est essentiellement le système racinaire qui représente l'interface de la plante avec la structure du sol. Cette dernière 
constitue un des enjeux de l'agronomie moderne (Henin et al., 1969), principalement dans des zones de culture intensive où la mécanisation a progressé anarchiquement et des zones où la mise en culture s'est étendue à des milieux aux conditions pédo-climatiques plus défavorables. Toutefois, si l'importance de la structure du sol est reconnue par une grande majorité d'agronomes et de praticiens, sa complexité rend son approche difficile. Ainsi, la caractérisation classique de la structure à l'aide de différents critères physiques pris isolément ne renseigne que d'une manière partielle sur le comportement agronomique du sol.

Ces considérations nous ont amenés à une réflexion approfondie en vue de rédiger un "cahier des charges" en matière d'appréciation agronomique de la structure. Celui-ci fait l'objet d'un premier chapitre. La perméabilité à l'air dont la définition et la métrologie ont été développées dans un premier article (Hinsinger et Mettauer, 1989) apparaît comme un critère intéressant pour rendre compte de la sructure du sol. Son adéquation au "cahier des charges" tant aux plans conceptuel que technique fait l'objet des deux chapitres suivants.

\section{"Cahier des charges" pour une appréciation agronomique de la structure : inadéquation des méthodes existantes}

\section{Approches descriptive et fonctionnelle de la structure}

Pour être pertinent, le critère d'appréciation à retenir doit être synthétique, au même titre que la notion de structure elle-même, afin de rendre compte au maximum des effets des différents facteurs physiques en jeu. Face à cette première contrainte, les agronomes ont développé deux approches qui sont à la fois complémentaires et antinomiques.

La première représente une approche descriptive qui, seule, relève de la synthèse. Elle se fonde sur la description du profil cultural (Henin et al., 1969) qui met en évidence les traits morphologiques symptomatiques des pratiques culturales antérieures. A ce niveau, Manichon (1982) a conforté cette approche descriptive en définissant de façons plus précises et objectives toute une série d'états structuraux qu'il a pu relier à leur passé cultural. Son approche permet ainsi de fournir une semi-quantification de la structure à l'échelle de la parcelle, en déterminant les proportions relatives (en termes de surfaces mesurées sur le profil cultural) des différents états structuraux recensés. L'application de sa démarche a aussi permis à Tardieu (1984) de relier ces données structurales à des morphologies et densités racinaires.

La deuxième approche possible est fonctionnelle et relève plus d'une démarche analytique. Elle repose sur le choix de paramètres pertinents qui fournissent chacun une vue quantitative mais partielle de la structure. Cette démarche explique leur intérêt fondamental en physique en sol, plus contestable en agronomie : la plante est le reflet de l'ensemble des facteurs au travers desquels la structure agit. Les deux variables les plus fréquemment mesurées (Henin, 1976) sont la porosité (densitométrie, humidimétrie et tensiométrie) et la résistance mécanique à la pénétration (pénétrométrie).

Pour ce qui concerne la porosité, il convient d'insister sur le fait que tout le volume poral mesuré n'influence pas les racines d'une manière identique. En particulier, la géométrie des pores est au moins aussi importante que leur volume total seul pris en compte (Scott Russel, 1977). Par ailleurs, le pénétromètre classiquement utilisé pour mesurer une résistance mécanique à la pénétration fournit, de par sa rigidité, une très mauvaise simulation du comportement des racines dans le sol (Baver et al., 1972) : celles-ci contournent les obstacles structuraux, plutôt que de les traverser.

L'intérêt de ces propriétés physiques, prises individuellement, est donc restreint, du moins dans l'optique d'une investigation de la structure finalisée par la connaissance des modalités de colonisation racinaire. L'emploi des méthodes classiques de mesure de ces paramètres peut ainsi conduire à un certain nombre d'inadéquations d'ordre conceptuel en matière d'appréciation agronomique de la structure du sol.

\section{Forte variabilité spatiale de la structure, au champ}

La très forte variabilité spatiale de la structure du sol est notamment subordonnée aux pratiques culturales : passage des roues et des divers outils dans différentes directions de circulation et avec différents angles d'entrure. Pour lui faire face, les agronomes ont fait appel à des méthodes cartographiques intéressantes : cartographie des états structuraux (Manichon, 1982) et des résistances mécaniques à la pénétration (Billot, 1982).

Toutes ces méthodes se caractérisent par une lourdeur de mise en œuvre qui limite notablement leur utilisation. La première, nécessitant l'ouverture d'une fosse et la description précise du profil cultural par un agronome compétent, représente un coût élevé en temps et en maind'œuvre. La seconde, si elle a l'avantage de se 
faire depuis la surface, souffre d'un coût rédhibitoire en matériel.

A l'échelle des îlots de culture hétérogènes, il convient d'orienter les recherches vers des méthodes de mesures plus rapides et de plus faible coût, autorisant la répétition des mesures.

\section{Forte variabilité temporelle de la structure de I'horizon cultivé}

Sous l'effet des remaniements fréquents dus aux interventions culturales, à l'action du climat et à celle des racines (et autres facteurs biotiques), la structure de l'horizon cultivé est une grandeur éminemment variable dans le temps. Face à cette évolution rapide, les méthodes précédemment évoquées s'avèrent peu adaptées, en raison de leur caractère destructif (cartographie des états structuraux, mesures de porosité) et/ou de leur lourdeur d'application (cartographie des états structuraux, cartographie pénétrométrique).

II convient donc ici encore, d'orienter les recherches vers des méthodes permettant des mesures de surface, suivant un protocole non ou peu destructif. A ce sujet, la pénétrométrie paraît être une technique satisfaisante pour suivre la variation temporelle de la structure, bien qu'elle n'autorise pas rigoureusement la répétition des mesures en un même point du champ (impact du pénétromètre).

\section{Intérêt d'ordre conceptuel de la perméabilité à l'air pour apprécier la structure et ses effets sur l'enracinement}

L'appréciation agronomique de la structure du sol est finalisée par la connaissance de ses effets sur la production végétale et, en particulier, sur le système racinaire ( $c f$. Introduction). La pertinence d'un critère d'appréciation de la structure se juge donc, tout d'abord, au travers des possibilités qu'il offre de rendre compte au maximum de ces effets.

Ainsi, sur le plan conceptuel, la perméabilité à l'air est un critère qui permet d'apprécier au mieux la circulation des fluides, d'une part et la fraction continue de la porosité d'autre part. Elle conduit donc tout naturellement à deux des facteurs physiques au travers desquels la structure agit sur la production végétale : aération et humidité.

In fine, il paraît important d'évaluer un tel critère d'appréciation agronomique de la structure, du fait de son aptitude à rendre compte de modalités de colonisation racinaire révélatrices de l'état structural du sol. II faut toutefois garder à l'esprit que la structure peut avoir des répercussions sur la production végétale à d'autres niveaux que celui du système racinaire.

\section{Perméabilité à l'air et circulation des fluides}

De par sa définition même, la notion de perméabilité relève de la théorie de l'écoulement des fluides en milieu poreux. II est donc naturel de relier la perméabilité à l'air aux possibilités d'écoulement en masse de l'air dans le sol. Mais, telle qu'elle a été définie (Hinsinger et Mettauer, 1989), il s'agit d'une perméabilité intrinsèque, c'est-à-dire indépendante du fluide considéré, lorsqu'elle est mesurée en sol sec. En toute rigueur, elle devrait donc être égale à la perméabilité à l'eau en sol saturé, dans l'hypothèse où le milieu poreux n'est pas modifié ni par l'air, ni par l'eau, lors de l'écoulement. Si l'air peut être considéré comme un fluide inerte vis-à-vis des constituants du sol, cela n'est pas le cas de l'eau : de par sa tension superficielle et les phénomènes de gonflement qu'elle induit (en présence d'argiles), l'eau modifie quelque peu la structure du milieu poreux lors de son imbibition. Dans cette optique, il apparaît délicat d'employer la perméabilité à l'eau comme caractéristique globale de la structure du sol.

Cette différence de comportement entre l'air et l'eau vis-à-vis des constituants du sol a conduit Reeve (1965) à définir le rapport $K_{\text {air }}[\mathrm{sol} \mathrm{sec}] /$ $K_{\text {eau }}$ [sol saturé ] (toujours $\geq 1$ ) comme un indice possible d'instabilité structurale. Ce critère est pertinent si la saturation du sol en eau a été obtenue dans des conditions empêchant l'apparition de phénomènes d'occlusion d'air, qui entraînent également la diminution de la perméabilité à l'eau par rapport à la perméabilité à l'air. En effet, ces phénomènes empêchent la saturation complète de la porosité par la phase mouillante. Par suite, la perméabilité à l'eau à "saturation" est toujours inférieure, parfois largement, à la perméabilité intrinsèque qui est bien estimée par la perméabilité à l'air en sol sec (la dessication complète étant facilement réalisable) (Hinsinger et Mettauer, 1989).

Toutefois, certaines études en parallèle des perméabilités à l'air et à l'eau, en fonction de la porosité remplie d'air, ont montré une bonne corrélation entre ces deux grandeurs, en milieu non saturé (Hartge, 1967; Rahte et Hartge, 1983). Dans ces travaux, la perméabilité à l'air permet d'approcher la description des transferts d'eau dans le sol (écoulement convectif). Or, ceux-ci constituent un phénomène essentiel à prendre en compte (modélisation des flux hydriques, application au drainage). A ce niveau, la mesure de la perméabilité à l'air peut apparaître comme une simplification méthodologique possible 
(mesure plus rapide). Toutefois, l'intérêt de son emploi est limité par la faible profondeur d'investigation possible : restriction à l'horizon de surface, rarement limitant au plan hydraulique. Son application dans les études de drainage est donc peu appropriée.

Par contre, l'écoulement convectif de l'air dans le sol est un phénomène qui ne contribue que faiblement au renouvellement de l'atmosphère du sol. L'aération relève, en effet, essentiellement du phénomène de diffusion gazeuse (Vilain, 1963). Toutefois, quel que soit le phénomène d'échange gazeux, il a lieu dans la fraction continue de l'espace poral exempt d'eau. Selon Russell (1973), la forme des pores continus affecte davantage la perméabilité à l'air, que le taux de diffusion lié plutôt au volume de cette fraction porale continue. Selon cet auteur, la perméabilité à l'air serait donc un critère peu pertinent pour préjuger de l'aération du sol. Toutefois, CollisGeorge (1953) affirme que, lorsque la porosité est faible, le processus de diffusion de l'air et, par conséquent, l'aération du sol, sont fortement corrélés à la perméabilité à l'air du sol.

En résumé, la perméabilité à l'air reste un bon critère pour préjuger de l'écoulement en masse de l'air dans le sol. Elle permet aussi une assez bonne approximation des phénomènes d'écoulement convectif de l'eau. Toutefois, son intérêt pour la connaissance de l'aération du sol est plus contestable en raison de la faible participation de ces phénomènes convectifs au renouvellement de l'atmosphère du sol. Ce jugement est néanmoins à moduler en fonction de la porosité du sol dont il convient d'étudier les relations avec la perméabilité.

\section{Perméabilité à l'air et porosité}

Lors des premières études sur la perméabilité à l'air, les auteurs ont souvent cherché à établir, implicitement (De Leenheer, 1953) ou explicitement (Hartge, 1968), des relations entre cette grandeur et la porosité. Ainsi, De Leenheer assimile, quelque peu abusivement, la notion de perméabilité à celle de macroporosité. Steinbrenner (1959) effectue un calibrage de son appareil de mesure en établissant une régression linéaire jugée satisfaisante $(r=-0,88$ sur 68 échantillons) entre la résistance au passage de l'air et "espace poral macroscopique" (fraction de l'espace poral rempli d'air à une tension d'eau de $1 / 3$ atm). II gradue, ainsi, directement son manomètre en pourcentage espace poral à partir de l'équation de régression et il exprime toutes ses mesures de perméabilité en terme de porosité.

Hartge (1968) réalise la première étude approfondie des relations entre perméabilité à l'air et porosité grâce à des mesures effectuées, cette fois-ci au laboratoire. II étudie, en fait, les relations existant avec différentes fractions de l'espace poral $(<30,30-50,50-150$ et $>150 \mu \mathrm{m})$, définies par des tensions d'eau variables. II montre que la relation entre perméabilité à l'air et volume poral est nette seulement pour les fractions porales les plus fines $(20-30$ et $30-50 \mu \mathrm{m})$. II explique l'absence de corrélation dans les fractions grossières par l'irrégularité de la géométrie de l'espace poral : tortuosité et variations de diamètre apparaissent ici nettement plus importante que dans les fractions fines. Dans ces dernières, l'adéquation avec le modèle capillaire (Gras, 1969) est meilleure et la loi de Hagen-Poiseuille applicable (Hubert, 1978). Dans la réalité, les formalismes de Poiseuille, puis de Kozeny-Carman qui permettent de relier perméabilité et porosité ne s'appliquent pas aux milieux poreux anisotropes et hétérogènes que sont les sols (Ball et al., 1981; Marshall, 1958). Cela explique le choix de ne pas les développer d'avantage ici, les références de Gras et de Hubert citées plus haut étant jugées suffisantes en la matière.

Hartge est ainsi l'un des premiers à souligner l'importance de la forme des pores - voire sa prépondérance par rapport à leur volume - dans la détermination de la perméabilité. II souligne, en effet, que les pores en doigt de gant, a fortiori ceux qui sont orientés dans un sens contraire à celui de l'écoulement, ne peuvent participer à celui-ci. La perméabilité dépend donc bien plus de la géométrie des seuls pores continus que de leur volume (Gras, 1969; Hanus, 1968). De ce fait, les relations univoques entre porosité et perméabilité à l'air apparaissent sujettes à caution, hormis le cas de petits échantillonnages de sols homogènes. A ce titre, Howard et Singer (1981) ont obtenu une mauvaise corrélation entre les valeurs de densité apparente (soit de porosité totale) mesurées sur mottes, et la perméabilité à l'air mesurée avec l'appareil de Steinbrenner (1959), contredisant ainsi les postulats de ce dernier.

Gupta et Bhatia (1975) estiment, pour leur part, que la perméabilité à l'air est sous la dépendance de la porosité remplie d'air (volume) quand la teneur en eau du sol est élevée, alors que c'est bien plus la distribution de tailles des pores qui apparaît déterminante à des humidités plus faibles. Ces conclusions sont en accord avec celles de Russell (1973) et Collis-George (1953) précédemment citées.

L'absence de relation étroite entre les deux variables, perméabilité à l'air et porosité, n'enlève rien à l'intérêt d'apprécier la première. La perméabilité à l'air semble être, en effet, le seul moyen possible pour l'investigation de la porosité 
continue, jugée ainsi, à la fois, en termes de volume et de géométrie (sans qu'il soit possible de faire la part de leurs rôles respectifs). Cette possibilité est d'autant plus intéressante, que l'importance de cette fraction de la porosité dans les phénomènes de transferts de fluides et pour la pénétration des racines dans le sol est grande.

\section{Perméabilité à l'air et colonisation racinaire}

En matière de colonisation racinaire de l'espace poral, deux écoles s'opposent actuellement. Les uns, à la suite d'Aubertin et Kardos (1965) considèrent que les racines ne colonisent que les pores préexistants, d'un diamètre supérieur ou égal au leur. Les autres, conformément aux travaux de Wiersum (1957), affirment que cette dernière hypothèse n'est valable qu'en milieu rigide, condition rarement rencontrée in situ : des sols riches en argile peuvent s'y rattacher lors de faibles teneurs en eau (Cockroft et al., 1969). Dans les autres cas, les racines peuvent exercer des contraintes mécaniques suffisantes pour créer la porosité nécessaire à leur pénétration ou agrandir les pores préexistants.

$\mathrm{Si}$, dans la nature, ces deux modes de colonisation semblent coexister, il est largement admis que les racines empruntent les chemins préférentiels que sont les pores continus préexistants et qui opposent les plus faibles résistances mécaniques (Scott Russel, 1977; et Ehlers et al., 1983). A ce niveau, la perméabilité à l'air apparaît comme un critère très pertinent pour l'appréciation de la structure en liaison avec la colonisation racinaire. Parallèlement, il semble nécessaire de recourir à d'autres critères comme la résistance mécanique à la pénétration ou la densité apparente pour mieux caractériser le degré de colonisation racinaire des zones à porosité défavorable (mottes, zones compactées). Cette double démarche a été tentée récemment par Groenevelt et al. (1984) avec succès.

L'intérêt de la perméabilité à l'air pour préjuger des effets de la structure du sol sur les modalités d'enracinement avait été pressenti par Kmoch en 1962. Ce chercheur montra, grâce à des mesures parallèles de densités racinaires et de diverses grandeurs physiques réalisées sur des carottages, que la perméabilité à l'air était le facteur physique le plus discriminant des états structuraux du sol; elle permettait, en effet, d'expliquer une large part des variations de densité racinaire. La même année, Phillips et Kirkham (1962) affirment que la perméabilité à l'air est un bon critère pour caractériser l'état de compacité du sol (davantage que son aération) et, par suite, la colonisation racinaire (en terme de densité racinaire). Au même titre que Kmoch (1962), ces auteurs insistent sur l'intérêt d'étudier conjointement les différentes propriétés physiques du sol (densité apparente, humidité, résistance mécanique et perméabilité à l'air) afin d'expliquer au mieux l'ensemble des modalités de colonisati - racinaire observées. Ces conclusions vont donc tout à fait dans le sens de la complémentarité des deux écoles évoquées au début de ce paragraphe.

Dans l'optique d'une appréciation de la structure du sol finalisée par la connaissance des modalités de colonisation racinaire, l'ntérêt conceptuel de la perméabilité à l'air est donc indiscutable, à la condition : 1) de restreindre cette connaissance à certaines modalités particulières où les racines primaires empruntent les voies préférentielles que sont les pores continus préexistants; 2) de compléter cette approche d'une mesure d'autres critères tels que la résistance mécanique à la pénétration (Groenevelt et al., 1984) pour rendre compte de toutes les modalités possibles.

Enfin, cet intérêt conceptuel se double d'un intérêt d'ordre technique qui devient d'autant plus important que se développent, parallèlement, de nouvelles techniques d'investigation non destructives des systèmes racinaires (Bohm, 1979; Maertens et al., 1982; Manantsoa, 1984).

\section{Intérêt d'ordre technique de la perméabilité à l'air pour rendre compte de la variabilité de la structure}

\section{Perméabilité à l'air et variabilité spatiale horizon- tale de la structure}

Les divers appareillages évoqués dans l'article I (Hinsinger et Mettauer, 1989) autorisent tous la réalisation rapide de nombreuses mesures de perméabilité à l'air. Celles-ci peuvent être largement valorisées, dans l'optique d'apprécier la variabilité spatiale horizontale de cette grandeur - et, par voie de conséquence, de certaines modalités d'enracinement - au travers de méthodes de traitement cartographique des données.

Ainsi Green et Fordham (1975) ont pu mettre en évidence l'effet de tassement des bandes de roulement, grâce à une représentation cartographique simplifiée de la perméabilité à l'air. En outre, ce traitement cartographique peut aussi faire l'objet de calculs mathématiques sophistiqués, relevant de la géostatistique, déjà appliquée, par ailleurs, aux données hydrodynamiques (Vauclin, 1982; Voltz, 1986).

En résumé, la représentation cartographique de la variabilité spatiale de la perméabilité à l'air 
devrait trouver un débouché certain dans la quantification d'accidents structuraux de surface, tels que la battance en particulier.

\section{Perméabilité à l'air et variabilité spatiale verticale de la structure}

Ces déterminations peuvent s'effectuer suivant deux protocoles distincts.

Le premier consiste à réaliser des mesures à différentes profondeurs, soit le long d'un profil dans une fosse (Green et Fordham, 1975), soit le long d'une carotte prélevée, de plusieurs décimètres (Kmoch, 1962). Dans les deux cas, la technique mise en œuvre présente le désavantage de nécessiter une excavation du sol et de ne fournir qu'une mesure de la perméabilité horizontale plutôt que verticale. Toutefois, le dernier inconvénient peut être levé en réalisant une excavation successive des couches supérieures consécutivement à la mesure de leur perméabilité.

En outre, l'avantage de ce protocole est d'autoriser la réalisation des mesures aux profondeurs voulues, sans aucune limitation autre qu'économique (coût des carottages profonds).

Le second protocole consiste à réaliser les mesures de perméabilité à l'air depuis la surface, sur des tranches de sol de plus en plus épaisses, grâce à un enfoncement progressif du cylindre de mesure. Un inconvénient de cette méthode résulte de la faiblesse de la profondeur d'investigation possible ( $\neq 40 \mathrm{~cm}$ supérieurs). Toutefois, ce protocole représente une simplification technique et économique notable qui explique l'intérêt porté actuellement par nous sur la question (travaux en cours).

\section{Perméabilité à l'air et variabilité temporelle de la structure}

Un autre intérêt important de la perméabilité à l'air réside dans la possibilité de réaliser sa mesure in situ suivant un protocole relativement peu destructif en tenant compte de toutes les limitations méthodologiques que cela comporte (Hinsinger et Mettauer, 1989).

La finalité d'un tel protocole non destructif réside dans le suivi de l'évolution de la structure du sol. Or, il apparaît difficile d'éviter toute dégradation de celle-ci lors des différentes phases d'extraction et d'enfoncement nécessaires à la réalisation des répétitions de mesures. Le palliatif méthodologique le plus intéressant, à ce sujet, consiste à laisser en place le cylindre de mesure pendant toute la durée de la campagne de mesure (Green et Fordham, 1975; Mettauer, travaux en cours). II peut toutefois se développer des fis- sures préférentielles induites par le cylindre qui faussent ainsi l'évolution normale de la structure. Outre ce problème majeur, cette démarche est limitée par la difficulté de comparer différentes mesures réalisées dans le temps, dans des conditions hydriques éminemment variables. Compte tenu de la forte dépendance de la perméabilité à l'air eu égard à la teneur en eau du sol, il faudrait réaliser les mesures successives dans des conditions hydriques similaires et/ou connues afin de rendre pertinente leur comparaison.

\section{Conclusion}

Compte tenu de la complexité de la notion de la structure du sol étudiée dans une optique agronomique, ainsi que des variabilités spatiale et temporelle, au champ, le cahier des charges pour les choix d'un critère pertinent de caractérisation de la structure est particulièrement contraignant. Cela explique la relative inadéquation des méthodes existantes, dont les plus performantes sont aussi les plus lourdes à mettre en œuvre (cartographie des états structuraux de Manichon, 1982). Ces faits vont, assurément, à l'encontre du cahier des charges que nous nous sommes fixés, en vue d'étudier la variabilité de la structure. Par contre, la perméabilité à l'air apparaît comme un critère répondant bien à ce cahier des charges, à différents niveaux.

Au plan conceptuel, il apparaît aussi que la perméabilité à l'air est un critère tout-à-fait pertinent pour préjuger des possibilités de colonisation préférentielle par les racines de la porosité continue préexistante. II s'avère, toutefois, judicieux de lui adjoindre des mesures de résistance mécanique à la pénétration (Groenevelt et al., 1984) afin de rendre compte de l'ensemble des modalités de colonisation racinaire possibles. Cela est d'autant plus envisageable que la micropénétrométrie répond assez bien au cahier des charges.

Au plan technique, la répétition possible des mesures dans l'espace et dans le temps (rapidité de mise en œuvre) permet une investigation, à faible coût, des variabilités spatiale et temporelle de la structure du sol (Green et Fordham, 1975). A ce niveau, la limitation essentielle n'est pas d'ordre technique mais méthodologique. En effet, la pertinence de la comparaison de différentes mesures entre elles suppose leur réalisation dans des conditions hydriques similaires. A ce niveau, le choix d'une humidité - standard de mesure de la perméabilité à l'air - s'impose. II paraît, a priori, intéressant de se placer à une humidité proche de la capacité au champ, pour différentes raisons. D'une part, il s'agit d'un état 
hydrique, relativement stable et facile à caractériser. D'autre part, il correspond à l'occupation des pores les plus fins (microporosité < 10 à $30 \mu \mathrm{m}$ ) par de l'eau; or, cette porosité est, dans tous les cas, inaccessible à la colonisation préférentielle par les racines (> $30 \mu \mathrm{m}$, très généralement) que permet, précisément, de prévoir la perméabilité à l'air. Avant de poursuivre toute investigation dans ce domaine, il paraît intéressant d'orienter les recherches actuelles sur la perméabilité à l'air dans cette voie.

\section{Rétérences}

Aubertin G.M. \& Kardos L.T. (1965) Root growth through porous media under controlled conditions. Effect of pore size and rigidity Soil Sci. Soc. Am. Proc. 29, 290-293

Ball B.C., Harris W. \& Burford J.R. (1981) A laboratory method to measure gas diffusion and flow in soil and other porous materials. Soil Sci. 32, 323-333

Baver L.D., Gardner W.H. \& Gardner W.R., (1972) Soil Physics 4th ed., John Wiley \& Sons, New York

Billot J.F. (1982) Les applications agronomiques de la pénétrométrie à l'étude de la structure des sols travaillés. Sci. Sol 3, 187-202

Blanchet R., Bosc M., Maertens C. \& Puech J. (1972) Root system transpiration and ions movement in the soil. Proceedings of the 9th Colloquium of the international Potash Institute, pp. 181-188

Bohm W. (1979) Methods of studying root systems. Springer-Verlag, Berlin

Cockroft B., Barley K.P. \& Greacen E.L. (1969) The penetration of clays by fine probes and root tips. Aust. J. Soil Res. 7, 333-348

Collis-George N. (1953) Relationship between air and water permeabilities in porous media. Soil Sci. 76, 239-250

De Leenheer L. (1953) La perméabilité du sol à l'air et la production en betteraves sucrières. Rev. Agric. 9, 1155-1161

Eavis B.W. (1972) Soil physical conditions affecting seedling root growth. I. Mechanical impedance, aeration and moisture availability as influenced by bulk density and moisture levels in a sandy loam soil. Plant Soil 36, 613-622

Ehlers W., Kopke V., Hesse F. \& Bohm W. (1983) Penetration resistance and root growth of oats in tilled and untilled loess soil. Soil Tillage Res. 3, 261-275

Gras R. (1969) Considerations sur la porosité et l'étude des milieux poreux Bull. Assoc. Fr. Etude Sol Sci. Sol 2, 79-89

Green R. D. \& Fordham S.J. (1975) A field method for determining air permeability in soil $\mathrm{In}$ : Soil Physical Conditions and Crop Production Tech. Bull. $29 \mathrm{HMSO}$, 273-288

Groenevelt P.H., Kay B.D. \& Grant C.D. (1984) Physical assessment of a soil with respect to rooting potential. Geoderma 34, 101-114

Gupta R.K. \& Bhatia S.C. (1975) Dependence of air permeability of black clay soil on moisture content and bulk density. J. Indian Soc. Soil Sci. 23, 280-285
Hanus H. (1968) Permeabilitätsmessungen mit Luft und Wasser an Modellproben. Z. Pflanzenernähr. Düng. Bodenkd. 120, 131-140

Hartge K.H. (1967) Der Zusammenhang zwischen Luft und Wasserpermeabilität in Bodenproben. Z. Planzenernähr. Düng. Bodenkd. 117, 97-107

Hartge K.H. (1968) Luftpermeabilität verschiedener Porenbereiche. Z. Pflanzenernähr. Düng. Bodenkd. $120,31-45$

Henin S., Gras R. \& Monnier G. (1969) Le profil cultural. L'état physique du sol et les conséquences agronomiques. Masson, Paris,

Henin S. (1976) Cours de Physique du Sol. ORSTOM - Editest, Bruxelles, 2 volumes

Hinsinger P. \& Mettauer H. (1989) De l'application de la perméabilité à l'air du sol en agronomie I. Notion de perméabilité à l'air. Théorie et Méthodologie. Agronomie 9, 3-12

Howard R.F. \& Singer M.J. (1981) Measuring forest soil bulk density using irregular hole, paraffin clod and air permeability Forest Sci. 27, 316-322

Hubert A. (1978) Relations entre la conductivité hydraulique et la structure des milieux poreux. Ann. Agron. 29, 475-498

Kmoch H.G. (1962) Die Luftdurchlässigkeit des Bodens. Ihre Bestimmung und ihre Bedeutung für einige ackerbauliche Probleme. Gebrüder Bornträger, Berlin pp. 90

Maertens C. \& Clauzel Y. (1982) Premières observations sur l'utilisation de l'endoscopie dans l'étude de l'enracinement in situ de plantes cultivées Sorghum vulgare et Lolium multiflorum. Agronomie, 2, 677-680

Manantsoa V. (1984) Conception et réalisation d'un endoscope automatique ou rhizoscope pour l'étude de la dynamique racinaire des plantes in situ. Thèse $3^{\text {e }}$ cycle, Electronique, Université de Mulhouse

Manichon H. (1982) Influence des systèmes de culture sur le profil cultural : élaboration d'une méthode de diagnostic basée sur l'observation morphologique. Thèse, Paris

Marshall T.J. (1958) A relationship between permeability and size distribution of pores. Soil Sci. 9, 1-8

Phillips R.E. \& Kirkham D. (1962) Soil compaction in the field and corn growth. Agron. J. 54, 29-34

Rahte I. \& Hartge K.H. (1983) Der Zusammenhang zwischen Luft- und Wasserleittähigkeit in nicht- gesättigten Boden. Mitt. Dtsch. Bodenkundt. Gesellsch. 38, 129-134

Reeve R.C. (1965) Air-to-water permeability ratio. $J$. Am. Soc. Agron. 9, 520-531

Russel M.B. (1949) Methods for measuring soil structure and aeration. Soil Sci. 68, 25-35

Russell E.W. (1973) Soil conditions and plant growth. 10 th ed. Longman, London

Scott Russel R. (1977) Plant root systems. Their function and interaction with the soil. McGraw-Hill, London,

Steinbrenner E.C. (1959) A portable air permeameter for forest soils. Soil Sci. Soc. Am. Proc. 23, 478-481

Tardieu F. (1984) Etude au champ de l'enracinement du maïs. Influence de l'état structural sur la répartition des racines, conséquences sur l'alimentation hydrique. Thèse Doct. Ing. INA-PG

Vauclin M. (1982) Méthodes d'études de la variabilité spatiale des propriétés d'un sol. INRA Publ. : les Col- 
loques de I'INRA, 15, 9-43

Vilain M. (1963) L'aération du sol. Ann. Agron. 14, 967-

998

Voltz M. (1986) Variabilité spatiale des propriétés phy- siques sur sol en milieu alluvial. Thèse Docteur-Ingénieur, ENSA : : untpellier

Wiersum L.K. (1957) The relationship of the size and structural rigidity of pores to their penetration by roots Plant Soil, 9, 75-85 